\title{
Understanding Inflections and Steps in Carbon Dioxide Adsorption Isotherms in Metal-Organic Frameworks
}

\author{
Krista S. Walton ${ }^{1}$, Andrew R. Millward ${ }^{2}$, David Dubbeldam ${ }^{3}$, Houston Frost ${ }^{3}$, \\ John J. Low ${ }^{4}$, Omar M. Yaghi ${ }^{5}$, and Randall Q. Snurr ${ }^{3 *}$ \\ ${ }^{1}$ Department of Chemical Engineering, Kansas State University, Manhattan, Kansas 66506 \\ ${ }^{2}$ Department of Chemistry, University of Michigan, Ann Arbor, Michigan 48109-1055 \\ ${ }^{3}$ Department of Chemical and Biological Engineering, Northwestern University \\ Evanston, Illinois 60208-3120 \\ ${ }^{4}$ UOP LLC, a Honeywell Company, 25 E. Algonquin Rd., Des Plaines, Illinois 60017 \\ ${ }^{5}$ Department of Chemistry and Biochemistry, University of California, Los Angeles, \\ Los Angeles, California 90095-1569
}

\section{Supporting Information}

\section{Details of GCMC Simulations}

The MOFs and carbon dioxide molecules were represented in atomistic detail. $\mathrm{CO}_{2}-\mathrm{CO}_{2}$ interactions were modeled using the TraPPE potential. ${ }^{1}$ This model places a Lennard-Jones center on each carbon and oxygen atom, a point charge of +0.70 on the center of mass of the carbon atom, and a charge of -0.35 on each oxygen atom to simulate the molecule's quadrupole moment. The $\mathrm{C}-\mathrm{O}$ bond length is $1.16 \AA$, and the bond angle is $180^{\circ}$. The Lennard-Jones parameters for the framework atoms were taken from the DREIDING force field. ${ }^{2}$ LorentzBerthelot mixing rules were employed to calculate sorbate/framework parameters. LennardJones interactions beyond $12.8 \AA$ were neglected. All Lennard-Jones parameters are given in Table S1.

No charges were used for the MOF frameworks. GCMC simulations which included $\mathrm{CO}_{2}-\mathrm{CO}_{2}$ electrostatic interactions agreed very well with experimental isotherms, and inclusion of framework charges did not provide any significant improvement to warrant the expensive Ewald calculations. For the $\mathrm{CO}_{2}-\mathrm{CO}_{2}$ Coulomb interactions, a cutoff based on the center-of-mass distance was used. This is justified (instead of the more expensive Ewald summation) because the $\mathrm{CO}_{2}-\mathrm{CO}_{2}$ quadrupole-quadrupole interaction is not long ranged.

Simulations were performed using the Music code. ${ }^{3}$ For each point on the isotherm, 30 million Monte Carlo steps were performed. Each step consisted of insertion of a new molecule, deletion of an existing molecule, or translation of an existing molecule. ${ }^{4}$ Typically, the first half of the run was used for equilibration, and the last half was used to calculate the ensemble averages.

The DREIDING forcefield was chosen for this work based upon its use in previous GCMC simulations of adsorption in MOFs. DREIDING has been shown to successfully reproduce adsorption isotherms for systems such as methane/IRMOFs ${ }^{5}$ and hydrogen/IRMOFs ${ }^{6}$. Also, the model works well in reproducing heats of adsorption for hydrogen in the IRMOF materials. ${ }^{6}$ In this work, we found very good agreement between simulations and experiment for $\mathrm{CO}_{2}$ in IRMOFs using this model. 
Table S1. Lennard-Jones Parameters Used in GCMC Simulations

\begin{tabular}{l|ll} 
Atom Pair & $\sigma(\AA)$ & $\varepsilon / \mathrm{k}_{\mathrm{b}}(\mathrm{K})$ \\
\hline $\mathrm{C}-\mathrm{C}$ & 2.80 & 27.0 \\
$\mathrm{O}-\mathrm{O}$ & 3.05 & 79.0 \\
$\mathrm{C}-\mathrm{O}$ & 2.93 & 36.0 \\
$\mathrm{C}-\mathrm{C}_{\mathrm{MOF}}$ & 3.32 & 35.96 \\
$\mathrm{C}-\mathrm{H}_{\mathrm{MOF}}$ & 2.82 & 14.38 \\
$\mathrm{C}-\mathrm{O}_{\mathrm{MOF}}$ & 2.92 & 36.07 \\
$\mathrm{C}-\mathrm{Zn}_{\mathrm{MOF}}$ & 3.42 & 27.35 \\
$\mathrm{O}-\mathrm{C}_{\mathrm{MOF}}$ & 3.26 & 61.51 \\
$\mathrm{O}-\mathrm{H}_{\mathrm{MOF}}$ & 2.95 & 24.59 \\
$\mathrm{O}-\mathrm{O}_{\mathrm{MOF}}$ & 3.04 & 61.70 \\
$\mathrm{O}-\mathrm{Zn}_{\mathrm{MOF}}$ & 3.55 & 46.78 \\
\hline
\end{tabular}

Note: $\mathrm{C}_{\mathrm{MOF}}$ and $\mathrm{O}_{\mathrm{MOF}}$ are carbon and oxygen framework atoms. These have different force field parameters than the carbon and oxygen atoms in the $\mathrm{CO}_{2}$ molecules.

Adsorption loadings obtained from GCMC simulations were converted from absolute loadings to excess loadings for comparison with experimental adsorption isotherms following the procedure given in Frost et al. ${ }^{6}$ Gas-phase fugacities for carbon dioxide were calculated using the PengRobinson equation of state. Absolute adsorption loadings were converted to adsorbed-phase densities using the crystal structure free volumes. These values in $\mathrm{cm}^{3} / \mathrm{g}$ are $1.315,2.615$, and 4.413 for IRMOF-1, -10 , and -16 , respectively. ${ }^{6}$

\section{Details of MOF Synthesis and Adsorption Measurements}

MOF syntheses and adsorption experimental details for $\mathrm{CO}_{2}$ isotherms at $298 \mathrm{~K}$ can be found in the cited literature. ${ }^{7}$

For sub-ambient temperature $\mathrm{CO}_{2}$ sorption experiments, a large batch of IRMOF-1 was prepared following a published procedure. ${ }^{8}$ The supernatant from the as-synthesized IRMOF-1 was decanted and the crystals were rinsed with dimethylformamide $(3 \times 10 \mathrm{~mL})$. The damp material was then immersed in $10 \mathrm{~mL} \mathrm{CHCl}_{3}$ to exchange out the solvent guests, and the $\mathrm{CHCl}_{3}$ was refreshed thrice in three days.

Isotherms were collected manually on a previously-reported ${ }^{9}$ discontinuous gravimetric sorption apparatus with a Cahn C-1000 microgravimetric balance ( $1 \mu \mathrm{g}$ sensitivity) and two MKS Baratron 622 A pressure transducers (10 and 1000 torr, accuracy $\pm 0.25 \%$ of range) enclosed in a glass-ware system. An amount of damp, $\mathrm{CHCl}_{3}$-exchanged IRMOF-1 was estimated so as to target an evacuated sample mass of 400 to $600 \mathrm{mg}$. The sample was loaded with minimal exposure to air into a tared cylindrical quartz sample bucket (30 mm height, $18 \mathrm{~mm}$ diameter, $4 \mathrm{~g}$ weight), and then mounted on the balance and enclosed in the system. 
To activate the damp sample in situ, the system pressure was reduced slowly to avoid elutriation ("bumping") until the sample mass was constant (within $0.05 \mathrm{mg}$ ) at room temperature and full vacuum $\left(<10^{-3}\right.$ torr $)$. To obtain sub-ambient temperature $\mathrm{CO}_{2}$ isotherms the system temperature was monitored by a thermocouple suspended in close proximity to the sample bucket while the sample chamber was bathed in various slushes: acetone/dry ice $(195 \mathrm{~K})$, chloroform/ $\mathrm{LN}_{2}(208$ $\mathrm{K})$, acetone/water/dry ice $(218 \mathrm{~K})$, acetonitrile $/ \mathrm{LN}_{2}(233 \mathrm{~K})$ and ice water $(273 \mathrm{~K})$. Attempts to carefully maintain both the level and consistency of the baths were achieved by occasional stirring and addition of cryogen.

To obtain each isotherm, the initial weight of the sample under vacuum was recorded after applying the isotherm bath. The $\mathrm{CO}_{2}$ (Bone Dry) adsorbate was added incrementally and data points were recorded when no further change in mass was observed $(<0.05 \mathrm{mg} / \mathrm{min})$. An empirical buoyancy correction was applied to all data points based on the weight change of standard aluminum foil weights within the analyte gas.

Table S2. $\mathrm{CO}_{2}$ Adsorption and Desorption Data for IRMOF-1 at 195K

\begin{tabular}{rrr|rrr}
$\mathbf{P}_{\text {eq }}$ (torr) & $\mathbf{W t}_{\text {buoy }}(\mathbf{m g})$ & $\mathbf{N}(\mathbf{m g} / \mathbf{g})$ & $\mathbf{P}_{\text {eq }}$ (torr) & $\mathbf{W t}_{\text {buoy }}(\mathbf{m g})$ & $\mathbf{N}(\mathbf{m g} / \mathbf{g})$ \\
\hline 0 & 171.5 & 0.0 & 751 & 426.9 & 1489.0 \\
12 & 176.0 & 26.3 & 700 & 426.8 & 1488.2 \\
40 & 183.1 & 67.5 & 600 & 425.5 & 1480.9 \\
74 & 191.7 & 117.6 & 511 & 423.3 & 1467.9 \\
104.8 & 200.7 & 169.9 & 341 & 414.9 & 1418.8 \\
116.2 & 205.0 & 195.2 & 260 & 409.7 & 1388.4 \\
125.3 & 208.5 & 215.7 & 197 & 400.5 & 1335.0 \\
135.6 & 213.8 & 246.8 & 180 & 397.5 & 1317.3 \\
145 & 229.4 & 337.3 & 160 & 392.4 & 1287.8 \\
150 & 315.4 & 838.7 & 150 & 384.4 & 1241.0 \\
155 & 386.4 & 1252.8 & 146 & 320.4 & 867.8 \\
165 & 391.4 & 1282.1 & 143 & 240.4 & 401.4 \\
175 & 394.4 & 1299.7 & 136 & 216.3 & 261.3 \\
190 & 397.5 & 1317.4 & 125 & 210.3 & 226.2 \\
215 & 402.5 & 1346.9 & 110 & 203.3 & 185.2 \\
250 & 407.6 & 1376.6 & 90 & 196.2 & 144.1 \\
301 & 410.8 & 1394.9 & 29 & 181.1 & 55.7 \\
350 & 413.9 & 1413.1 & 0 & 173.0 & 8.6 \\
450 & 419.1 & 1443.7 & & & \\
547 & 422.4 & 1462.6 & & & \\
654 & 424.7 & 1475.9 & & & \\
751 & 426.9 & 1489.0 & & & \\
\hline
\end{tabular}


Table S3. $\mathrm{CO}_{2}$ Adsorption and Desorption Data for IRMOF-1 at 208K

\begin{tabular}{rrr|rrr}
$\mathbf{P}_{\text {eq }}$ (torr) & $\mathbf{W t}_{\text {buoo }}(\mathbf{m g})$ & $\mathbf{N}(\mathbf{m g} \mathbf{g})$ & $\mathbf{P}_{\text {eq }}$ (torr) & $\mathbf{W t}_{\text {buoy }}(\mathbf{m g})$ & $\mathbf{N}(\mathbf{m g} / \mathbf{g})$ \\
\hline 0 & 171.2 & 0.0 & 750.2 & 411.7 & 1405.5 \\
10.7 & 173.4 & 13.3 & 696 & 408.8 & 1388.4 \\
54.7 & 180.1 & 52.5 & 550 & 401.4 & 1345.3 \\
92.6 & 186.7 & 91.1 & 375 & 391.0 & 1284.3 \\
121.4 & 191.8 & 120.7 & 293 & 381.7 & 1230.5 \\
142.2 & 195.9 & 144.4 & 285 & 380.7 & 1224.5 \\
156.9 & 198.3 & 158.6 & 275 & 378.7 & 1212.7 \\
190.5 & 205.8 & 202.4 & 255 & 280.6 & 639.8 \\
238.1 & 223.4 & 305.3 & 240 & 228.6 & 335.7 \\
249.1 & 234.3 & 369.2 & 175 & 202.4 & 182.9 \\
266 & 280.7 & 639.9 & 75 & 184.2 & 76.2 \\
273.7 & 365.7 & 1136.7 & 0 & 172.0 & 5.0 \\
300.1 & 380.8 & 1224.7 & & & \\
336.5 & 386.9 & 1260.3 & & & \\
362.3 & 388.9 & 1272.4 & & & \\
427.5 & 393.1 & 1296.7 & & & \\
510.3 & 399.3 & 1333.0 & & & \\
605.6 & 404.5 & 1363.7 & & & \\
750.2 & 411.7 & 1405.5 & & & \\
\hline
\end{tabular}

Table S4. $\mathrm{CO}_{2}$ Adsorption and Desorption Data for IRMOF-1 at $218 \mathrm{~K}$

\begin{tabular}{rrr|rrr}
$\mathbf{P}_{\text {eq }}$ (torr) & $\mathbf{W t}_{\text {buov }}(\mathbf{m g})$ & $\mathbf{N}(\mathbf{m g} / \mathbf{g})$ & $\mathbf{P}_{\text {eq }}$ (torr) & $\mathbf{W t}_{\text {buov }}(\mathbf{m g})$ & $\mathbf{N}(\mathbf{m g} / \mathbf{g})$ \\
\hline 0 & 171.5 & 0.0 & 750 & 382.9 & 1232.4 \\
30 & 174.1 & 14.9 & 713 & 378.8 & 1208.6 \\
102 & 181.3 & 56.8 & 580 & 365.5 & 1130.8 \\
145 & 185.4 & 80.7 & 542 & 356.4 & 1077.8 \\
204 & 191.5 & 116.6 & 510 & 336.3 & 960.7 \\
276 & 200.7 & 170.1 & 490 & 279.2 & 628.1 \\
347 & 210.9 & 229.5 & 455 & 229.2 & 336.0 \\
402 & 220.0 & 282.8 & 358 & 213.9 & 247.1 \\
448 & 229.1 & 335.9 & 257 & 198.7 & 158.2 \\
485 & 261.2 & 523.1 & 177 & 189.5 & 104.5 \\
503 & 304.3 & 774.0 & 75 & 178.2 & 38.9 \\
525 & 347.3 & 1025.0 & & & \\
535 & 355.4 & 1071.8 & & & \\
550 & 361.4 & 1107.0 & & & \\
601 & 368.5 & 1148.6 & & & \\
642 & 371.6 & 1166.7 & & & \\
688 & 376.8 & 1196.5 & & & \\
750 & 382.9 & 1232.4 & & & \\
\hline
\end{tabular}


Table S5. $\mathrm{CO}_{2}$ Adsorption and Desorption Data for IRMOF-1 at $233 \mathrm{~K}$

\begin{tabular}{rrr|rrr}
$\mathbf{P}_{\mathbf{e q}}$ (torr) & $\mathbf{W t}_{\text {buoy }}(\mathbf{m g})$ & $\mathbf{N}(\mathbf{m g} \mathbf{g})$ & $\mathbf{P}_{\text {eq }}$ (torr) & $\mathbf{W t}_{\text {buoy }}(\mathbf{m g})$ & $\mathbf{N}(\mathbf{m g} / \mathbf{g})$ \\
\hline 0 & 191.1 & 0.0 & 735.8 & 248.2 & 298.7 \\
11.9 & 191.8 & 3.8 & 675 & 238.7 & 249.3 \\
52.3 & 194.1 & 15.6 & 548 & 226.4 & 184.8 \\
91.5 & 196.3 & 27.3 & 300 & 208.8 & 92.5 \\
125.6 & 198.2 & 37.4 & 195.3 & 202.2 & 58.0 \\
147.3 & 199.4 & 43.7 & & & \\
154.5 & 199.9 & 46.1 & & & \\
196.1 & 202.4 & 59.1 & & & \\
240 & 205.1 & 73.4 & & & \\
353.7 & 212.3 & 111.3 & & & \\
491.5 & 222.8 & 166.1 & & & \\
603.9 & 232.0 & 214.3 & & & \\
735.8 & 248.2 & 298.7 & & & \\
\hline
\end{tabular}

Table S6. $\mathrm{CO}_{2}$ Adsorption and Desorption Data for IRMOF-1 at $273 \mathrm{~K}$

\begin{tabular}{rrr|rrr}
$\mathbf{P}_{\text {eq }}$ (torr) & $\mathbf{W t}_{\text {buoy }}(\mathbf{m g})$ & $\mathbf{N}(\mathbf{m g} \mathbf{g})$ & $\mathbf{P}_{\text {eq }}(\mathbf{t o r r})$ & $\mathbf{W t}_{\text {buoy }}(\mathbf{m g})$ & $\mathbf{N}(\mathbf{m g} / \mathbf{g})$ \\
\hline 0 & 191.1 & 0.0 & 748.4 & 203.7 & 66.1 \\
12 & 191.3 & 1.2 & 424.8 & 198.1 & 36.8 \\
50.4 & 191.9 & 4.4 & 163.1 & 193.8 & 14.3 \\
91.2 & 192.6 & 8.1 & 0 & 191.1 & 0.0 \\
126.4 & 193.2 & 11.0 & & & \\
144.6 & 193.5 & 12.5 & & & \\
153.7 & 193.7 & 13.5 & & & \\
191.7 & 194.3 & 16.6 & & & \\
250.6 & 195.2 & 21.7 & & & \\
340 & 196.7 & 29.2 & & & \\
498.3 & 199.2 & 42.3 & & & \\
592.1 & 200.9 & 51.5 & & & \\
748.4 & 203.7 & 66.1 & & & \\
\hline
\end{tabular}


Figure S1. $\mathrm{CO}_{2}$ sorption isotherms for IRMOF-1 from 195 to 273K. Filled circles are adsorption; open circles are desorption.

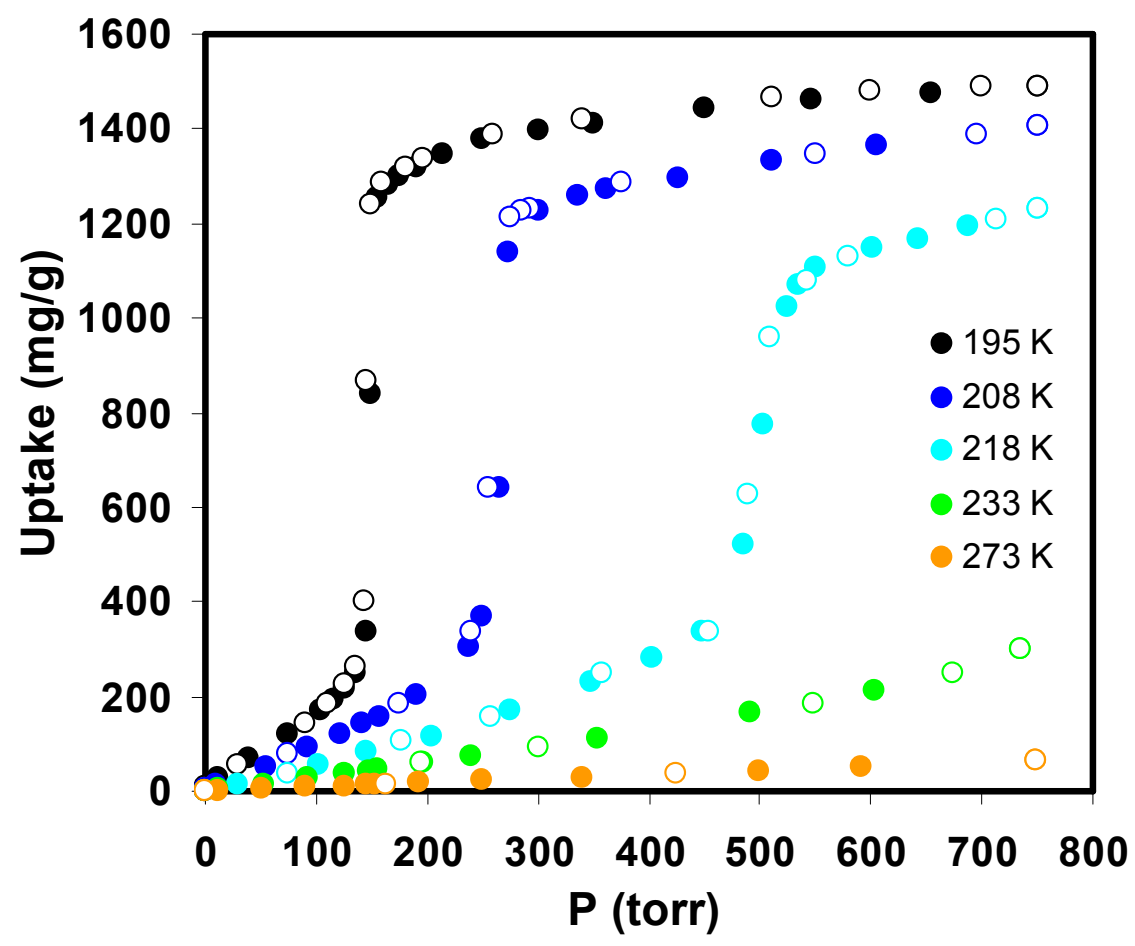


Table S7. $\mathrm{CO}_{2}$ Adsorption and Desorption Data for IRMOF-1 at $298 \mathrm{~K}$

\begin{tabular}{|c|c|c|c|c|c|}
\hline$P_{\text {eq }}$ (psia) & $\mathbf{W t}_{\text {buoy }}(\mathrm{mg})$ & $\mathrm{N}(\mathrm{mg} / \mathrm{g})$ & $\mathbf{P}_{\text {eq }}($ psia $)$ & $\mathbf{W t}_{\text {buoy }}(\mathrm{mg})$ & $\mathrm{N}(\mathrm{mg} / \mathrm{g})$ \\
\hline 0.0 & 24.05 & 0.00 & 612.9 & 47.34 & 968.19 \\
\hline 17.8 & 25.19 & 47.30 & 317.0 & 45.05 & 872.60 \\
\hline 30.1 & 26.00 & 81.03 & 168.3 & 38.03 & 581.11 \\
\hline 46.3 & 27.02 & 123.07 & 15.0 & 25.05 & 41.45 \\
\hline 60.1 & 27.97 & 162.85 & & & \\
\hline 77.0 & 29.24 & 215.75 & & & \\
\hline 92.1 & 30.48 & 267.22 & & & \\
\hline 104.3 & 31.54 & 311.34 & & & \\
\hline 119.0 & 32.95 & 369.87 & & & \\
\hline 136.3 & 34.72 & 443.26 & & & \\
\hline 150.9 & 36.24 & 506.46 & & & \\
\hline 166.5 & 37.78 & 570.77 & & & \\
\hline 178.6 & 38.84 & 614.66 & & & \\
\hline 195.0 & 40.12 & 667.69 & & & \\
\hline 209.2 & 41.05 & 706.44 & & & \\
\hline 224.9 & 41.90 & 741.85 & & & \\
\hline 240.7 & 42.65 & 773.13 & & & \\
\hline 255.2 & 43.25 & 797.86 & & & \\
\hline 269.4 & 43.71 & 817.05 & & & \\
\hline 285.4 & 44.19 & 836.97 & & & \\
\hline 299.2 & 44.56 & 852.34 & & & \\
\hline 313.7 & 44.85 & 864.69 & & & \\
\hline 347.2 & 45.52 & 892.29 & & & \\
\hline 372.8 & 45.89 & 907.80 & & & \\
\hline 404.7 & 46.28 & 923.98 & & & \\
\hline 436.0 & 46.60 & 937.04 & & & \\
\hline 463.8 & 46.82 & 946.28 & & & \\
\hline 494.6 & 47.00 & 953.89 & & & \\
\hline 524.7 & 47.13 & 959.23 & & & \\
\hline 554.3 & 47.25 & 964.27 & & & \\
\hline 584.0 & 47.32 & 967.02 & & & \\
\hline 612.9 & 47.34 & 968.19 & & & \\
\hline
\end{tabular}


Table S8. $\mathrm{CO}_{2}$ Adsorption and Desorption Data for IRMOF-3 at 298K

\begin{tabular}{rrr|rrr}
$\mathbf{P}_{\text {eq }}(\mathbf{p s i a})$ & $\mathbf{W t}_{\text {buoy }}(\mathbf{m g})$ & $\mathbf{N}(\mathbf{m g} / \mathbf{g})$ & $\mathbf{P}_{\text {eq }}(\mathbf{p s i a})$ & $\mathbf{W t}_{\text {buoy }}(\mathbf{m g})$ & $\mathbf{N}(\mathbf{m g} \mathbf{g})$ \\
\hline 0.0 & 43.82 & 0.00 & 612.9 & 80.19 & 829.91 \\
16.2 & 46.18 & 53.64 & 416.7 & 79.30 & 809.40 \\
30.1 & 48.36 & 103.55 & 216.6 & 74.67 & 703.95 \\
48.1 & 51.41 & 172.99 & 107.8 & 63.22 & 442.59 \\
61.0 & 53.71 & 225.62 & 14.3 & 45.99 & 49.35 \\
75.2 & 56.51 & 289.50 & 0.0 & 43.83 & 0.23 \\
90.1 & 59.49 & 357.47 & & & \\
105.5 & 62.51 & 426.30 & & & \\
121.0 & 65.22 & 488.18 & & & \\
135.2 & 67.48 & 539.86 & & & \\
148.9 & 69.21 & 579.21 & & & \\
164.3 & 70.86 & 616.82 & & & \\
180.1 & 72.20 & 647.59 & & & \\
194.1 & 73.17 & 669.72 & & & \\
208.3 & 74.07 & 690.05 & & & \\
224.8 & 74.88 & 708.69 & & & \\
239.5 & 75.50 & 722.83 & & & \\
255.4 & 76.11 & 736.66 & & & \\
269.5 & 76.57 & 747.09 & & & \\
284.3 & 76.98 & 756.60 & & & \\
298.3 & 77.34 & 764.66 & & & \\
315.1 & 77.73 & 773.69 & & & \\
343.4 & 78.23 & 785.00 & & & \\
376.8 & 78.75 & 797.04 & & & \\
406.0 & 79.12 & 805.45 & & & \\
432.8 & 79.43 & 812.39 & & & \\
462.2 & 79.64 & 817.35 & & & \\
504.3 & 79.94 & 824.21 & & & \\
523.6 & 79.99 & 825.17 & & & \\
554.0 & 80.10 & 827.73 & & & \\
581.6 & 80.16 & 829.19 & & & \\
612.9 & 80.19 & 829.91 & & & \\
\hline & & & & & \\
& & & & \\
& & & & \\
& & & & \\
& & & & \\
\end{tabular}


Table S9. $\mathrm{CO}_{2}$ Adsorption and Desorption Data for MOF-177 at 298K

\begin{tabular}{rrr|rrr}
$\mathbf{P}_{\text {eq }}(\mathbf{p s i a})$ & $\mathbf{W t}_{\text {buoy }}(\mathbf{m g})$ & $\mathbf{N}(\mathbf{m g} / \mathbf{g})$ & $\mathbf{P}_{\text {eq }}(\mathbf{p s i a})$ & $\mathbf{W t}_{\text {buoy }}(\mathbf{m g})$ & $\mathbf{N}(\mathbf{m g} / \mathbf{g})$ \\
\hline 0.0 & 16.01 & 0.00 & 616.0 & 39.91 & 1493.48 \\
14.9 & 16.57 & 35.13 & 314.4 & 36.71 & 1293.68 \\
31.4 & 17.26 & 78.55 & 212.5 & 30.77 & 922.56 \\
45.2 & 17.89 & 117.56 & 116.5 & 21.92 & 369.31 \\
59.1 & 18.53 & 157.41 & & & \\
76.0 & 19.37 & 210.11 & & & \\
89.9 & 20.14 & 258.00 & & & \\
106.0 & 21.11 & 318.84 & & & \\
120.4 & 22.04 & 377.14 & & & \\
135.0 & 23.13 & 444.79 & & & \\
149.7 & 24.33 & 520.01 & & & \\
165.9 & 25.80 & 611.52 & & & \\
179.9 & 27.13 & 695.22 & & & \\
195.8 & 28.70 & 793.23 & & & \\
209.7 & 30.08 & 879.19 & & & \\
225.7 & 31.64 & 976.49 & & & \\
239.4 & 32.90 & 1055.08 & & & \\
254.0 & 34.04 & 1126.59 & & & \\
270.1 & 34.96 & 1184.34 & & & \\
287.2 & 35.72 & 1231.85 & & & \\
299.7 & 36.21 & 1262.03 & & & \\
316.5 & 36.76 & 1296.58 & & & \\
343.4 & 37.51 & 1343.22 & & & \\
374.6 & 38.14 & 1383.04 & & & \\
402.9 & 38.61 & 1412.29 & & & \\
444.1 & 39.14 & 1444.99 & & & \\
463.6 & 39.30 & 1455.05 & & & \\
492.6 & 39.51 & 1468.60 & & & \\
523.7 & 39.70 & 1480.31 & & & \\
555.6 & 39.78 & 1485.41 & & & \\
582.8 & 39.89 & 1491.87 & & & \\
616.0 & 39.91 & 1493.48 & & & \\
\hline & & & & & \\
& & & & \\
& & & & \\
& & & & \\
& & & & \\
\end{tabular}


Figure S2. $\mathrm{CO}_{2}$ sorption isotherms for IRMOF-1, IRMOF-3 and MOF-177 at 298K. Filled circles are adsorption; open circles are desorption.

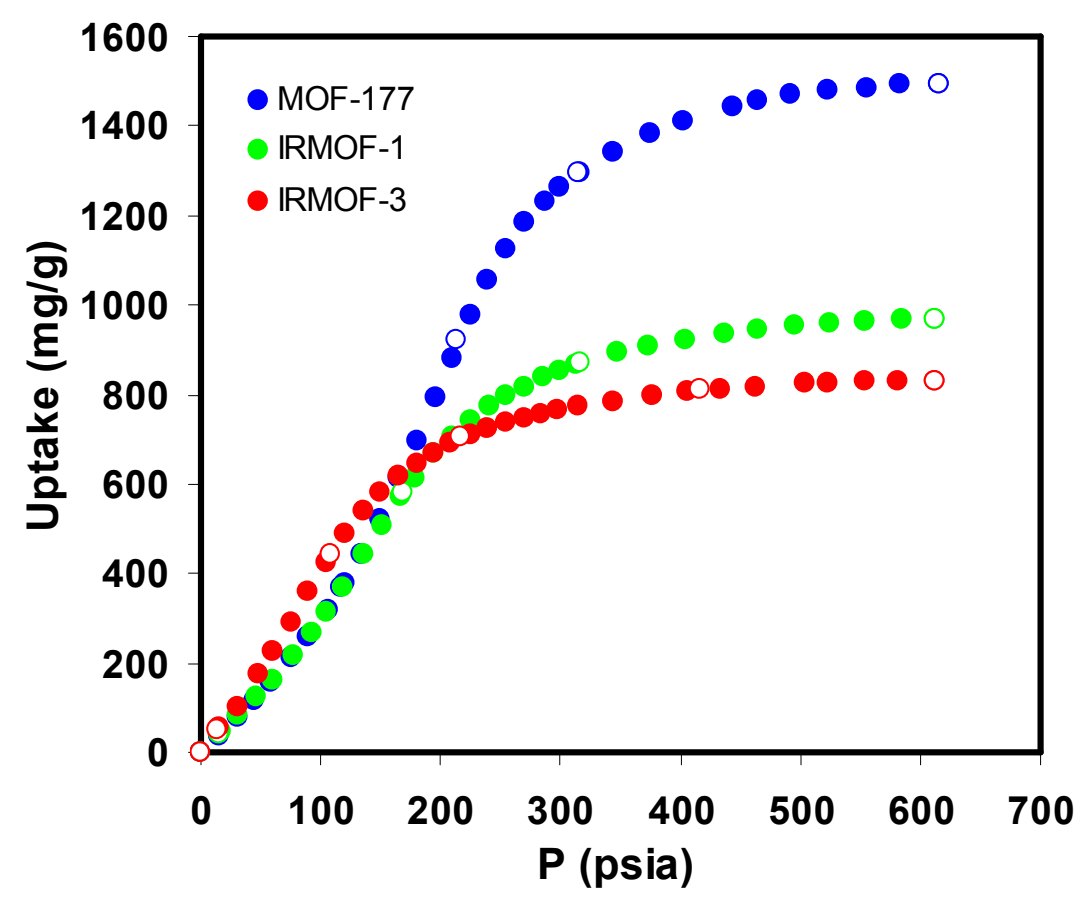

\section{References}

(1) Potoff, J.J.; Siepmann, J.I. AIChE J. 2001, 47, 1676.

(2) Mayo, S. L.; Olafson, B. D.; Goddard, W. A. J. Phys. Chem. 1990, 94, 8897.

(3) Gupta, A.; Chempath, S.; Sanborn, M.J.; Clark, L.A.; Snurr, R.Q. Molec. Simulation 2003, 29, 29.

(4) Frenkel, D.; Smit, B. Understanding Molecular Simulation: From Algorithms to Applications. $2^{\text {nd }}$ Ed., Academic Press: San Diego, 2002.

(5) Düren, T.; Sarkisov, L.; Yaghi, O.M.; Snurr, R.Q. Langmuir 2004, 20, 2683.

(6) Frost, H.; Düren, T.; Snurr, R.Q. J. Phys. Chem. B. 2006, 110, 9565.

(7) Millward, A.R.; Yaghi, O.M. J. Am. Chem. Soc. 2006, 127, 17998.

(8) Li, H.; Eddaoudi, M.; O'Keeffe, M.; Yaghi, O.M. Nature 1999, 402, 276.

(9) Eddaoudi, M.; Li, H. L.; Yaghi, O.M. J. Am. Chem. Soc. 2000, 122, 1391. 